\title{
Towards Robust Direct Perception Networks for Automated Driving
}

\author{
Chih-Hong Cheng
}

\begin{abstract}
We consider the problem of engineering robust direct perception neural networks with output being regression. Such networks take high dimensional input image data, and they produce affordances such as the curvature of the upcoming road segment or the distance to the front vehicle. Our proposal starts by allowing a neural network prediction to deviate from the label with tolerance $\Delta$. The source of tolerance can be either contractual or from limiting factors where two entities may label the same data with slightly different numerical values. The tolerance motivates the use of a non-standard loss function where the loss is set to 0 so long as the prediction-to-label distance is less than $\Delta$. We further extend the loss function and define a new provably robust criterion that is parametric to the allowed output tolerance $\Delta$, the layer index $\tilde{l}$ where perturbation is considered, and the maximum perturbation amount $\kappa$. During training, the robust loss is computed by first propagating symbolic errors from the $\tilde{l}$-th layer (with quantity bounded by $\kappa$ ) to the output layer, followed by computing the overflow between the error bounds and the allowed tolerance. The overall concept is experimented in engineering a direct perception neural network for understanding the central position of the ego-lane in pixel coordinates.
\end{abstract}

\section{INTRODUCTION}

Deep neural networks (DNNs) are increasingly used in the automotive industry for realizing perception functions in automated driving. The safety-critical nature of automated driving requires the deployed DNNs to be highly dependable. Among many dependability attributes we consider the robustness criterion, which intuitively requires a neural network to produce similar output values under similar inputs. It is known that DNNs trained under standard approaches can be difficult to exhibit robustness. For example, by imposing carefully crafted tiny noise on an input data point, the newly generated data point may enable a DNN to produce results that completely deviate from the originally expected output.

In this paper, we study the robustness problem for direct perception networks in automated driving. The concept of direct perception neural networks refers to learning affordances (high-level sensory features) [1]-[3] such as distance to lane markings or distance to the front vehicles, directly from high-dimensional sensory inputs. In contrast to classification where the output criterion for robustness is merely the sameness of the output label for input data under perturbation, direct perception commonly uses regression output. For practical systems, it can be unrealistic to assume that trained neural networks can produce output regression that perfectly matches the numerical values as specified in the labels.

Chih-Hong Cheng is with Corporate R\&D, DENSO AUTOMOTIVE Deutschland GmbH. c. cheng@denso-auto. de
Towards this issue, our proposal is to define tolerance $\Delta$ that explicitly regulates the allowed output deviation from labels. Pragmatically, the source of tolerance arises from two aspects, namely (i) the quality contract between car makers and their suppliers, or (ii) the inherent uncertainty in the manual labelling proces 11 We subsequently define a loss function that integrates the tolerance - the prediction error is set to be 0 so long as the prediction falls within the tolerance bound. Thus the training intuitively emphasizes reducing the worst case (i.e., to bring the prediction back to the tolerance). This is in contract to the use of standard loss functions (such as mean-squared-error) where the goal is to bring every prediction to be close to the label.

As robustness requires that input data being perturbed should produce results similar to input data without perturbation, $\Delta$ can naturally be overloaded to define the "sameness" of the regression output under perturbation. Based on this concept, we further propose a new criterion for provable robustness [4]-[10] tailored for regression, which is parametric to the allowed output tolerance $\Delta$, the layer index $\tilde{l}$ where perturbation is considered, and the maximum perturbation amount $\kappa$. The robust criterion requires that for any data point in the training set, by applying any feature-level perturbation on the $l$-th layer with quantity less than $\kappa$, the computation of the DNN only leads to slight output deviation (bounded by $\Delta$ ) from the associated ground truth. Importantly, the introduction of parameter $\tilde{l}$ overcomes scalability and precision issues, while it also implicitly provides capabilities to capture global input transformations (cf. Section $\square$ for a detailed comparison to existing work). By carefully defining the loss function as the interval overflow between (i) the computed error bounds due to perturbation and (ii) the allowed tolerance interval, the loss can be efficiently computed by summing the overflow of two endpoints in the propagated symbolic interval.

To evaluate our proposed approach, we have trained a direct perception network with labels created from publicly accessible datasets. The network takes input from road images and produces affordances such as the central position of the ego-lane in pixel coordinates. The positive result of our preliminary experiment demonstrates the potential for further applying the technology in other automated driving tasks that use DNNs.

(Structure of the Paper) The rest of the paper is struc-

\footnotetext{
${ }^{1}$ Our very preliminary experiments demonstrated that, when trying to label the center of the ego lane on the same image having 1280 pixels in width, a labelling deviation of 10 pixels for two consecutive trials is very common, especially when the labelling decision needs to be conducted within a short period of time.
} 


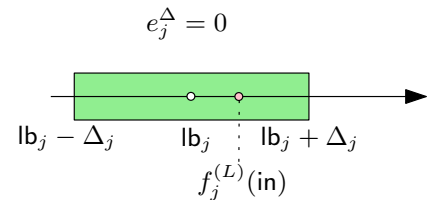

(a)

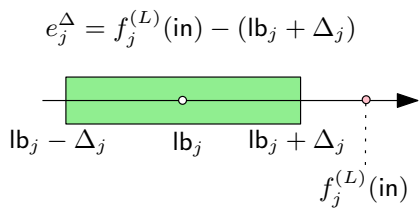

(b)
Fig. 1: Illustrating the error defined by $\Delta_{j}$.

tured as follows. Section II starts with basic formulations of neural networks and describes the tolerance concept. It subsequently details how the error between predictions and labels, while considering tolerance, can be implemented with GPU support. Section III extends the concept of tolerance for provable robustness by considering feature-level perturbation. Section IV details our initial experiment in a highway vision-based perception system. Finally, we outline related work in Section V and conclude the paper in Section VI with future directions.

\section{Neural Network AND TOLERANCE}

A neural network $\mathcal{N}$ is comprised of $L$ layers where operationally, the $l$-th layer for $l \in\{1, \ldots, L\}$ of the network is a function $g^{(l)}: \mathbb{R}^{d_{l-1}} \rightarrow \mathbb{R}^{d_{l}}$, with $d_{l}$ being the dimension of layer $l$. Given an input data point in $\in \mathbb{R}^{d_{0}}$, the output of the $l$-th layer of the neural network $f^{(l)}$ is given by the functional composition of the $l$-th layer and previous layers $f^{(l)}($ in $):=\circ_{i=1}^{(l)} g^{(i)}($ in $)=g^{(l)}\left(\ldots g^{(2)}\left(g^{(1)}(\right.\right.$ in $\left.\left.)\right)\right)$. $f^{(L)}$ (in) is the prediction of the network under input data point in. Throughout this paper, we use subscripts to extract an element in a vector, e.g., use $\mathrm{in}_{j}$ to denote the $j$-th value of in.

Given a neural network $\mathcal{N}$ following above definitions, let $\mathcal{D}_{\text {train }}:=\{(\mathrm{in}, \mathrm{lb})\}$ be the training data set, with each data point in $\in \mathbb{R}^{d_{0}}$ having its associated label $\mathrm{lb} \in \mathbb{R}^{d_{L}}$. Let $\left(\Delta_{1}, \ldots, \Delta_{d_{L}}\right)$, where $\forall j \in\left\{1, \ldots, d_{L}\right\}: \Delta_{j} \geq 0$, be the output tolerance. We integrate tolerance to define the error between a prediction $f^{(L)}$ (in) of the neural network and the label lb. Precisely, for output index $j \in\left\{1, \ldots, d_{L}\right\}$,

$e_{j}^{\Delta}\left(f_{j}^{(L)}(\mathrm{in}), \mathrm{lb}_{j}\right):= \begin{cases}0 & \text { if }\left|f_{j}^{(L)}(\mathrm{in})-\right| \mathrm{b}_{j} \mid \leq \Delta_{j} \\ \min \left(\left|f_{j}^{(L)}(\mathrm{in})-\left(\mathrm{lb}_{j}-\Delta_{j}\right)\right|\right. & \\ \left.\left|f_{j}^{(L)}(\mathrm{in})-\left(\mathrm{lb}_{j}+\Delta_{j}\right)\right|\right) & \text { otherwise }\end{cases}$

Figure 1 illustrates the intuition of such an error definition. We consider a prediction to be correct (i.e., error to be 0 ) when the prediction is within interval $\left[\mathrm{lb}_{j}-\Delta_{j}, \mathrm{lb}_{j}+\Delta_{j}\right]$ (Figure 1.a). Otherwise, the error is the distance to the boundary of the interval (Figure 1 1 b). Finally, we define the in-sample error to be the sum of squared error for each output dimension, for each data point in the training set.

Definition 1 (Interval tolerance loss): Define the error in the training set (i.e., in-sample error) to be $E_{\text {train }}^{\Delta}:=$
$\frac{1}{N} \sum_{(\text {in,lb }) \in \mathcal{D}_{\text {train }}}(E($ in, lb $))$, where $N=\left|\mathcal{D}_{\text {train }}\right|$ and $E($ in, lb $)=\sum_{j=1}^{d_{L}}\left(e_{j}^{\Delta}\left(f_{j}^{(L)}(\text { in }), \mathrm{lb}_{j}\right)\right)^{2}$.

One may observe that the loss function defined above is designed as an extension of the mean-squared-error (MSE) loss function.

Lemma 1: When $\forall j \in\{1, \ldots, L\}: \Delta_{j}=0$, $E_{\text {train }}^{\Delta}$ is equal to the mean-squared-error loss function $\frac{1}{N} \sum_{(\text {in,lb }) \in \mathcal{D}_{\text {train }}} \| f^{(L)}($ in $)-\mathrm{lb} \|^{2}$.

Proof: By setting $\Delta_{j}=0, e_{j}^{\Delta}\left(f_{j}^{(L)}(\mathrm{in}), \mathrm{lb}_{j}\right)$ can be simplified to $\mid f_{j}^{(L)}($ in $)-\left|\mathrm{b}_{j}\right|$. Thus, in Definition 1 , $E$ (in, lb) is simplified to $\sum_{j=1}^{d_{L}} \mid f_{j}^{(L)}$ (in) $-\left|\mathrm{lb}_{j}\right|^{2}$, which is equivalent to computing the square of the L2-norm $\| f^{(L)}$ (in) $-\mathbf{l b} \|^{2}$.

(Implementing the loss function with GPU support) For commonly seen machine learning infrastructures such as TensorFlow ${ }^{2}$ or PyTorch ${ }^{3}$, for training a neural network that uses standard layers such as ReLU [11], ELU [12], Leaky ReLU [13] as well as convolution, one only needs to manually implement the customized loss function, while back propagation capabilities for parameter updates are automatically created by the infrastructure. In the following, we demonstrate a rewriting of $e_{j}^{\Delta}()$ such that it uses built-in primitives supported by TensorFlow. Such a rewriting makes it possible for the training to utilize GPU parallelization.

Lemma 2 (Error function using GPU function primitives): Let $\operatorname{clip}_{\geq 0}(x)$ be a function that returns $x$ if $x \geq 0$; otherwise it returns 0 . Define $\hat{e}_{j}^{\Delta}\left(f_{j}^{(L)}(\mathrm{in}), \mathrm{Ib}_{j}\right)$ to be $\max \left(\operatorname{clip}_{\geq 0}\left(\left(\mathrm{lb}_{j}-\Delta_{j}\right)-f_{j}^{(L)}(\right.\right.$ in $\left.)\right), \operatorname{clip}_{\geq 0}\left(f_{j}^{(L)}(\right.$ in $)-\left(\mathrm{lb}_{j}+\right.$ $\left.\left.\Delta_{j}\right)\right)$ ). Then $e_{j}^{\Delta}\left(f_{j}^{(L)}(\mathrm{in}), \mathrm{lb}_{j}\right)=\hat{e}_{j}^{\Delta}\left(f_{j}^{(L)}(\mathrm{in}), \mathrm{lb}_{j}\right)$.

Proof: (Sketch) It can be intriguing to reason that $\hat{e}_{j}^{\Delta}()$ and $e_{j}^{\Delta}()$ are equivalent functions, i.e., the if-thenelse statement in $e_{j}^{\Delta}()$ is implicitly implemented using the clip $_{\geq 0}$ primitive ${ }^{4}$ in $\hat{e}_{j}^{\Delta}()$. To assist understanding, Figure 2 provides a simplified proof by enumerating all three possible cases regarding the relative position of $f_{j}^{(L)}$ (in) and the tolerance interval, together with their intermediate computations. Diligent readers can easily swap the constants in Figure 2 and establish a formal correctness proof.

\section{Provably Robust Training}

This section starts by outlining the concept of featurelevel perturbation, followed by defining symbolic loss. It then defines provably robust training and how training can be made efficient with GPU support. For simplifying notations, in this section let $v \oplus c$ be an operation that (1) if $c$ is a scalar, adds $c$ to every dimension of a vector $v$, or (2) if $c$ is a vector, perform element-wise addition.

Definition 2 (Output bound under ( $\tilde{l}, \kappa)$-perturbation): Given a neural network $\mathcal{N}$ and an input data point in, let

\footnotetext{
${ }^{2}$ Google TensorFlow: http://www.tensorflow.org

${ }^{3}$ Facebook PyTorch: https://www.pytorch.org

${ }^{4}$ The $\operatorname{clip}_{\geq 0}()$ function is implemented in Google TensorFlow using tf.keras.backend.clip.
} 


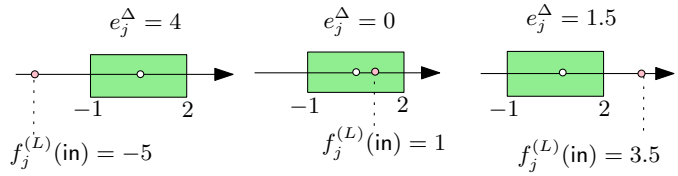

\begin{tabular}{|c|c|c|c|}
\hline$a:=\left(\mathrm{Ib}_{j}-\Delta_{j}\right)-f_{j}^{(L)}(\mathrm{in})$ & 4 & -2 & -4.5 \\
\hline$b:=f_{j}^{(L)}(\mathrm{in})-\left(\mathrm{Ib}_{j}+\Delta_{j}\right)$ & -7 & -1 & 1.5 \\
\hline$c:=\operatorname{clip}_{\geq 0}(a)$ & 4 & 0 & 0 \\
\hline$d:=\operatorname{clip}_{\geq 0}(b)$ & 0 & 0 & 1.5 \\
\hline$\hat{e}_{j}^{\Delta}:=\max (c, d)$ & 4 & 0 & 1.5 \\
\hline
\end{tabular}

Fig. 2: Illustrating the output equivalence between functions $e_{j}^{\Delta}()$ and $\hat{e}_{j}^{\Delta}()$ using examples.

$[L, U]$ be the output bound subject to $(\tilde{l}, \kappa)$-perturbation. For each output dimension $j \in\left\{1, \ldots, d_{L}\right\},\left[L_{j}, U_{j}\right]$, the $j$-th output bound of the neural network, satisfies the following condition: If $o_{j}=g_{j}^{(L)}\left(g^{(L-1)}\left(\ldots\left(g^{(\tilde{l})}(\mathrm{fv})\right)\right)\right)$ where $\mathrm{fv} \in\left[f^{(\tilde{l}-1)}(\right.$ in $) \oplus-\kappa, f^{(\tilde{l}-1)}($ in $\left.) \oplus \kappa\right]$, then $o_{j} \in\left[L_{j}, U_{j}\right]$.

Definition 2 can be understood operationally: first compute $f^{(\tilde{l}-1)}$ (in) which is the feature vector of in at layer $\tilde{l}$. Subsequently, try to perturb $f^{(\tilde{l}-1)}$ (in) with some noise bounded by $[-\kappa, \kappa]$ in each dimension, in order to create a perturbed feature vector fv. Finally, continue with the computation using the perturbed feature vector (i.e., $\left.g^{(L)}\left(g^{(L-1)}\left(\ldots\left(g^{(\tilde{l})}(\mathrm{fv})\right)\right)\right)\right)$, and the computed prediction in the $j$-th dimension should be bounded by $\left[L_{j}, U_{j}\right]$. Note that Definition 2 only requires $\left[L_{j}, U_{j}\right]$ to be an overapproximation over the set of all possible predicted values, as the logical implication is not bidirectional. The bound can be computed efficiently with GPU support via approaches such as abstract interpretation with boxed domain (i.e., dataflow analysis [14], [15]).

Given the output bound under $(\tilde{l}, \kappa)$-perturbation, our goal is to define a loss function that computes the overflow of output bounds over the range of tolerant values.

Definition 3 (Symbolic loss): For the $j$-th output of the neural network, for (in,lb) $\in \mathcal{D}_{\text {train }}$, Let $[L, U]$ be the output bound by feeding the network with in following Definition 2. Define $e_{j}^{\langle\Delta, \tilde{l}, \kappa\rangle}([L, U], \mathrm{lb})$, the symbolic loss for the $j$-th output subject to $(\tilde{l}, \kappa)$-perturbation with $\Delta$ tolerance, to be overflow $\left(\left[L_{j}, U_{j}\right],\left[\mathrm{lb}_{j}-\Delta_{j}, \mathrm{lb}_{j}+\Delta_{j}\right]\right)$. The function overflow $\left(I_{1}, I_{2}\right)$ equals $\sum_{m=1}^{n} \operatorname{dist}\left(\frac{1}{2}\left(L_{\alpha_{m}}+\right.\right.$ $\left.\left.U_{\alpha_{m}}\right),\left[\mathrm{lb}_{j}-\Delta_{j}, \mathrm{lb}+\Delta_{j}\right]\right)$ where

- $\left[L_{\alpha_{1}}, U_{\alpha_{1}}\right], \ldots,\left[L_{\alpha_{n}}, U_{\alpha_{n}}\right]$ are maximally disjoint intervals of $I_{1} \backslash I_{2}$.

- Function dist $(A,[B, C])$ computes the shortest distance between point $A$ and points in the interval $[B, C]$.

The intuition behind the defined symbolic loss is to (i) compute the intervals of the output bound that are outside the tolerance and subsequently, (ii) consider the loss as the accumulated effort to bring the center of each interval back to the tolerance interval. Figure 3 illustrates the concept.
- In Figure 3 a, as the both the output lower-bound and the upper-bound are contained in the tolerance interval, the loss is set to 0 .

- For Figure 3-d, the output bound is $[-2,5.5]$ while the tolerance interval is $[-1,4]$. Therefore, there are two maximally disjoint intervals $[-2,-1]$ and $[4,5.5]$ falling outside the tolerance. The loss is the distance between the center of each interval $[-2,-1],[4,5.5]$ to the tolerance boundary, which equals $\left|\frac{1}{2}(5.5+4)-4\right|+$ $\left|\frac{1}{2}(-2+(-1))-(-1)\right|=1.25$.

- Lastly in Figure 3 e, the complete interval $[5.5,9]$ is outside the tolerance interval. The loss is the distance between the center of the interval $\left(\frac{5.5+9}{2}\right)$ to the boundary, which equals $\frac{5.5+9}{2}-4=3.25$.

Definition 4 (Symbolic tolerance loss): Given a neural network $\mathcal{N}$, define the loss on the training set $\mathcal{D}_{\text {train }}$ to be $E_{\text {train }}^{(\Delta, \tilde{l}, \kappa)}=\frac{1}{N} \sum_{(\mathrm{in}, \mathrm{lb}) \in \mathcal{D}_{\text {train }}}(E(\mathrm{in}, \mathrm{lb}))$, where $E(\mathrm{in}, \mathrm{lb})=$ $\sum_{j=1}^{d_{L}}\left(e_{j}^{(\Delta, \tilde{l}, \kappa)}([L, U], \mathrm{lb})\right)^{2}, N=\left|\mathcal{D}_{\text {train }}\right|$, and $[L, U]$ is computed using Definition 2 .

(Implementing the loss function with GPU support) The following result states that computing the symbolic loss on the $j$-th output can be done very effectively by averaging the interval loss for $L_{j}$ and $U_{j}$, thereby further utilizing the result from Lemma 2 for efficient computation via GPU support.

Lemma 3 (Computing symbolic loss by taking end-points): For the $j$-th output of the neural network, for (in, lb) $\in \mathcal{D}_{\text {train }}$, the symbolic loss subject to $(\tilde{l}, \kappa)$ perturbation with $\Delta$ tolerance has the following property:

$$
e_{j}^{\langle\Delta, \tilde{l}, \kappa\rangle}([L, U], \mathrm{lb})=\frac{1}{2}\left(e_{j}^{\Delta}\left(L_{j}, \mathrm{lb}_{j}\right)+e_{j}^{\Delta}\left(U_{j}, \mathrm{lb}_{j}\right)\right)
$$

where $[L, U]$ is computed by feeding the network with in using Definition 2.

Proof: (Sketch) Here for simplicity, we illustrate in Figure 3 all 6 possible cases concerning the relative position between interval $\left[L_{j}, U_{j}\right]$ and interval $\left[\mathrm{lb}_{j}-\Delta_{j}, \mathrm{lb}_{j}+\Delta_{j}\right]$. For each case, results of computing $\frac{1}{2}\left(e_{j}^{\Delta}\left(L_{j}, \mathrm{lb}_{j}\right)+e_{j}^{\Delta}\left(U_{j}, \mathbf{l b}_{j}\right)\right)$ are shown directly in Figure 3 Readers can easily swap the constants in Figure 3 to create a formal correctness proof.

One immediate observation of Lemma 3 is that when the maximum allowed perturbation $\kappa$ equals 0 , no feature perturbation appears, and the output bound can be as tight as a single point $f^{(L)}\left(\right.$ in). Therefore, one has $L=U=f^{(L)}$ (in) and it enables the following simplification.

Lemma 4: When $\kappa=0$, values computed using symbolic loss can be the same as values computed from interval loss, i.e., $e_{j}^{\langle\Delta, \tilde{l}, \kappa\rangle}([L, U], \mathbf{l b})=e_{j}^{\Delta}\left(f_{j}^{(L)}(\mathrm{in}), \mathbf{l b}_{j}\right)$.

Results from Lemma 1, 3 and 4 altogether offer a pragmatic method for training. First, one can train a network using the loss function MSE; based on the chained rule of Lemma 1 and Lemma 3, using MSE loss is equivalent to the special case where $\Delta=0$ and $\kappa=0$. Subsequently, one can train the network with interval loss; it is equivalent to 


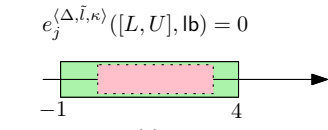

(a)
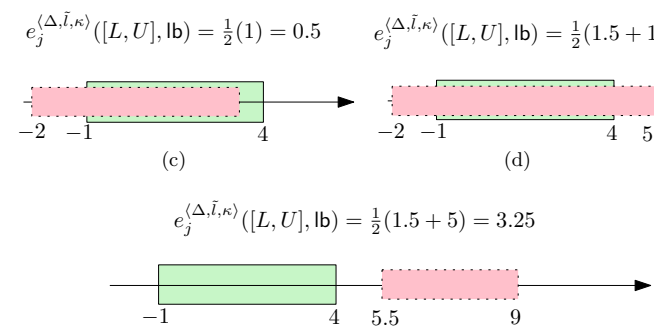

(e)

$e_{j}^{\langle\Delta, \tilde{l}, \kappa\rangle}([L, U], \mathrm{lb})=\frac{1}{2}(1+3)=2$

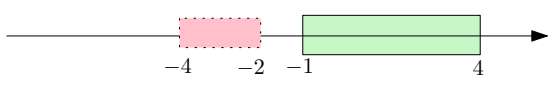

(f)

$\square$ Tolerance bound $\left[\mathrm{lb}_{j}-\Delta_{j}, \mathrm{lb}_{j}+\Delta_{j}\right]$
$\cdots \ldots \ldots$. Output bound $\left[L_{j}, U_{j}\right]$

Fig. 3: Computing symbolic interval loss

the special case where $\kappa=0$. Finally, one enlarges the value of $\kappa$ towards provably robust training.

Finally, we summarize the theoretical guarantee that the new training approach provides. Intuitively, the below lemma states that if there exists an input in' (not necessarily contained in the training data) whose feature vector is sufficiently close to the feature vector of an existing input in, then the output of the network under in' will be close to the output of the network under in.

Lemma 5 (Theoretical guarantee on provable training): Given a neural network $\mathcal{N}$ and $\mathcal{D}_{\text {train }}:=\{(\mathrm{in}, \mathrm{lb})\}$ be the training data set, if $E_{\text {train }}^{(\Delta, \tilde{l}, \kappa)}=0$, then for every input in', if exists an input training data (in, lb) $\in \mathcal{D}_{\text {train }}$ such that $f^{(\tilde{l}-1)}\left(\right.$ in') $\in\left[f^{(\tilde{l}-1)}(\right.$ in $) \oplus(-\kappa), f^{(\tilde{l}-1)}($ in $\left.) \oplus+\kappa\right]$, then $f^{(L)}($ in') $\in[\mathrm{l} \mathrm{b} \oplus(-\Delta), \mathrm{l} \mathbf{b} \oplus \Delta]$.

Proof: When $E_{\text {train }}^{(\Delta, \tilde{l}, \kappa)}=0$, from Definition 4 one knows that for every input data (in, lb) $\in \mathcal{D}_{\text {train }}$, the corresponding $e_{j}^{(\Delta, \tilde{l}, \kappa)}([L, U], \mathrm{lb})=0$. This implies that the output lowerbound $L_{j}$ and upper-bound $U_{j}$, computed using Definition 3 with input in, are contained in $\left[\mathrm{lb}_{j}-\Delta_{j}, \mathrm{lb}_{j}+\Delta_{j}\right]$.

In Definition 3 , the computation of $L_{j}$ and $U_{j}$ considers every point in $\left[f^{(l-1)}(\right.$ in $) \oplus(-\kappa), f^{(\tilde{l}-1)}($ in $\left.) \oplus+\kappa\right]$. Therefore, so long as $f^{(\tilde{l}-1)}\left(\right.$ in' $\left.^{\prime}\right) \in\left[f^{(l-1)}(\right.$ in $) \oplus(-\kappa), f^{(\tilde{l}-1)}($ in $\left.) \oplus+\kappa\right]$, the output of the neural network under in' should be within $[L, U]$, thereby within $[\mathrm{lb} \oplus(-\Delta), \mathrm{lb} \oplus \Delta]$.

As a consequence, if one perturbs a data point in in the training set to in ${ }^{\prime}$, so long as the perturbed input has produced similar high-level feature vectors at layer $\tilde{l}-1$, the output under perturbation is provably guaranteed to fall into the tolerance interval.

\section{EXPERIMENT}

To understand the proposed concept in a realistic setup, we engineered a direct perception network for identifying the center of the ego lane in $x$-position, by considering a fixed height $(y=500)$ in pixel coordinate ${ }^{5}$. For repeatability purposes, we take the publicly available TuSimple dataset for lane detection ${ }^{6}$ and create labels from its associated ground truth.

\section{A. Creating data for experimenting direct perception}

In the TuSimple lane detection dataset, labels for lane markings contain three parts:

- $L_{y}$ containing a list of $y$ coordinates that are used to represent a lane.

- A list of lanes $\mathcal{L}_{1}, \mathcal{L}_{2} \ldots$, where for each lane $\mathcal{L}_{i}$, it stores a list of $x$ coordinates.

- The corresponding image raw file.

Therefore, for the $i$-th lane in an image, its lane markings are $\left(L_{i}[0], L_{y}[0]\right),\left(L_{i}[1], L_{y}[1]\right),\left(L_{i}[2], L_{y}[2]\right)$, and so on. The lanes are mostly ordered from left to right, with some exceptions (e.g., files clips/0313-1/21180/20.jpg and clips/0313-2/550/20.jpg) where one needs to manually reorder the lanes.

We created a script to automatically generate affordance labels for our experiment: First, fix the height to be 500, followed by finding two adjacent lanes where (1) the first lane marking is on the left side of the image, and (2) the second lane marking is on the right side. If the script cannot find such two lanes, and the script just omits the data as it requires manual labelling. Subsequently, we take the average $x$-position of two such lanes to be the center of the ego lane (i.e., the label). Therefore, every output label is an integer between 0 and 720 . See Figure 4 for the ground truth of the lane marking and the generated center-of-ego-lane position (small green dot). Furthermore, we duplicate images whose created labels are far ( $\geq 100$ pixels) from the center of the image, to highlight the importance of rare events and to compensate the problem of not having enough labelled data.

For an image in the TuSimple dataset, it has a size of $1280 \times 720$. We crop the $y$-direction to keep only pixels with indices in range $[208,720)$, as the cropped elements are largely sky and cloud. Subsequently, resize the image by $\frac{1}{4}$ and make it grayscale. This ultimately creates, for each input image, a tensor of dimension $320 \times 128 \times 1$ (in TensorFlow, the shape of the tensor equals $(128,320,1))$. We also perform simple normalization using $t(v)=\frac{2}{255} v-1$ such that the value $v$ of each pixel, originally in the range $[0,255]$, is now in the interval $[-1,1]$.

\section{B. Evaluation}

In our experiment, we use a network architecture similar to the one shown in Figure 5 As a baseline, we train 20 networks using Xavier weight initialization [16], with each

\footnotetext{
${ }^{5}$ It is possible to train a network to produce multiple affordances. Nevertheless, in the evaluation, our decision to only produce one affordance is to clearly understand the impact of the methodology for robustness.

${ }^{6}$ TuSimple data set is available at: https://github.com/ TuSimple/tusimple-benchmark/wiki
} 

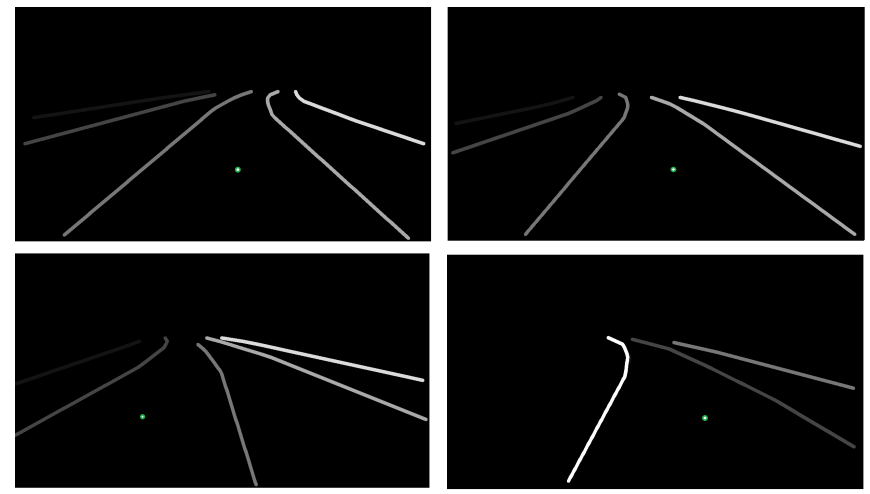

Fig. 4: Creating labels for the center of the ego-lane (green dot), from labels of lane markings (lines in the images) in the TuSimple data set.

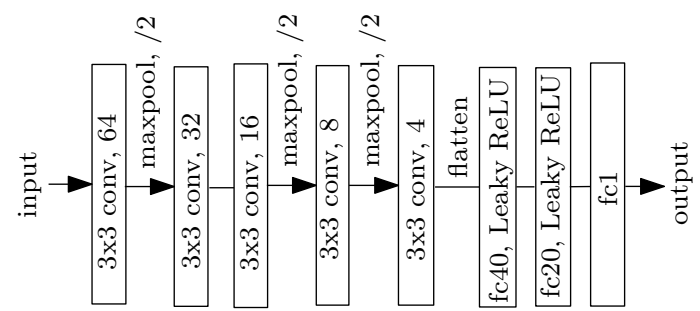

Fig. 5: The network architecture used in evaluation.

network starting with a unique random seed between 0 and 19. This is for repeatability purposes (via fixing random seeds) and for eliminating manual knowledge bias in summarizing our findings (via training many models). The training uses the Adaptive Moment Estimation (Adam) optimization algorithm [17] with learning rate $\alpha=0.01$ for 20 epochs and subsequently, $\alpha=0.001$ for another 10 epochs. Finally, we take the best 6 performed models and further apply robust training techniques, by using Adam optimization algorithm with $\alpha=0.001$ for yet another 10 epochs. In terms of average-case performance, the baseline model and the model further trained with robust loss have similar performance.

We use the single-step (non-iterative) fast gradient sign method (FGSM) [18] as the baseline perturbation technique to understand the effect of applying robust training. Precisely, we compare the minimum step size $\epsilon$ to make an originally perfect prediction (both for the standard network and the network further trained using robust loss) deviate with 80 pixels. As we use the single-step method, the parameter $\epsilon$ is directly related to the intensity of perturbation. Figure 6 shows the overall summary on each baseline model and its further (robustly) trained model. In some models (such as model 2), further training does not lead to significant improvement, as for these networks, the minimum $\epsilon$ values for enabling successful perturbations are largely similar. Nevertheless, for other models such as model 1 or model 6, a huge portion of the images require larger $\epsilon$ in the robustly trained model, in order to successfully create the adversarial effect. Figure 7 details the required $\epsilon$ value for successful attacks in model 1, where each image is a point in the

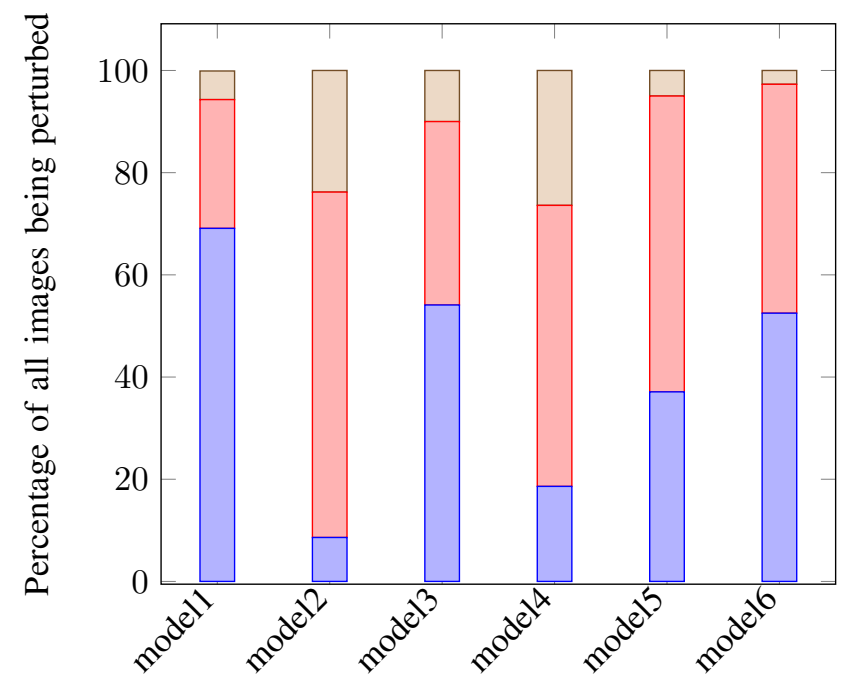

$\square$ Robust training has larger $\epsilon \square$ roughly equal $\square$ MSE has larger $\epsilon$

Fig. 6: Comparing performance on perturbation, where "roughly equal" refers to the case where the difference of $\epsilon$ values for two models are smaller than 0.05 .

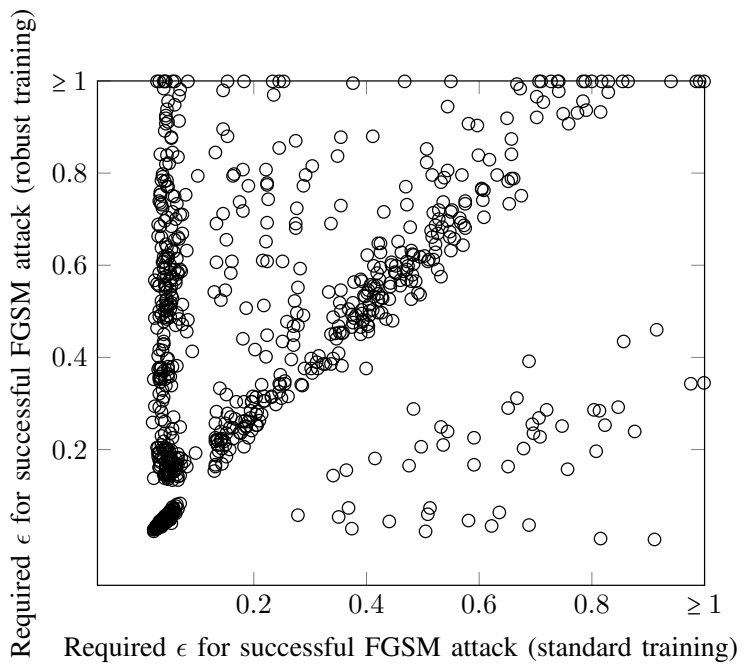

Fig. 7: Comparing models trained using standard (MSE) and symbolic loss approaches (for applying $\Delta=10$ and $\kappa=0.01$ on the layer $f_{C} 40$ of Figure 5 .

coordinate plane. One immediately observes that the majority of the points are located at the top-left of the coordinate plane, i.e., one requires larger amount of perturbation for models under robust training to reach the desired effect.

Although our initial evaluation has hinted promises, it is important to understand that a more systematic analysis, such as evaluating the technique on multiple data sets and a deeper understanding over the parameter space of $\Delta, \kappa$, and $\tilde{l}$, is needed to make the technology truly useful.

\section{RELATED WORK}


For engineering robust neural networks, the concept in this paper is highly related to the work of provably robust training [4]-[10]. Compared to existing work, the key difference lies in two aspects: (i) Existing work focuses on classification, while we focus on output regression for learning affordances. This is made possible by considering an afore-specified tolerance interval. (ii) Existing work perturbs directly on the individual input channels (which is a special case of ours by setting $\tilde{l}=1$ ), while our definition of layer index $\tilde{l}$ generally applies to close-to-output layers. This implies a feature-level perturbation rather than perturbation on individual bits (which makes existing methods hard to characterize global transformation such as image distortion). It also increases size of the network that can be trained: As symbolic bound propagation starts at the $\tilde{l}$-th layer, the execution time and the precision for bound propagation is indifferent to the depth of convolution layers before feature layers. In this work, we only use the boxed domain to compute the over-approximation, following the work of [15]. One can also use more precise abstract-interpretation domains such as Zonotope [19] with a price of increasing the training time, but pragmatically (due to the loss never approaching 0) the imprecision of boxed domain can be compensated by simply using smaller $\kappa$ values.

The concept of provable robustness training by definition only establishes a proof on every data point used in training. Therefore, even for perfectly trained neural networks, it is still insufficient to argue quality assurance on data points in the Operating Design Domain (ODD) that are distant from the training data. It may be complemented by recent research attempts in testing, formal verification, or the introduction of systematic processes for dependable machine learning. Testing techniques, as demonstrated in recent research trend of finding adversarial examples (due to excessive results in this direction, we refer readers to a recent survey paper [20] for existing approaches), largely use gradient-based search techniques to find small perturbation around original inputs that makes the output of neural network behave erroneously. It focuses on finding counter-examples rather than providing a guaranteed proof for the absence of undesired behavior. Some recently proposed test metrics aim to provide a pragmatic argument of completeness of testing [21][24] mimicking the coverage criteria (e.g., line coverage) appeared in classical software testing techniques. Formal verification [15], [19], [25]-[38] views the neural network as a mathematical object and performs symbolic analysis either via SMT [26], [28], abstract interpretation [19], [25], [33], [36], MILP [15], [29], or specialized search techniques [27], [30]-[32], [34], [35]. Overall, formal verification offers strong promise on the absence undesired behaviors, but the scalability to very deep networks with inputs from highdimensional pixel images remains limited.

\section{CONCLUding REMARKS}

In this paper, we considered the problem of engineering robust networks for direct perception, where regression rather than classification is used for output. We defined a loss function by integrating the practically-driven tolerance concept, thereby guiding the training process with the goal of bringing predictions back to the tolerance interval rather than a stricter form of being close to labels. Extending the tolerance concept by integrating perturbation, we created conditions where one can ascertain robustness with provably guarantees. The proposed loss functions are proven to be generalizations of the MSE loss function commonly used in standard training approaches.

The inability to create a systematic approach for engineering robust neural networks for perception systems is one of the most critical barriers towards safe automated driving. We believe that the approach suggested in this paper, in particular the tolerance-driven approach for machine learning, offers an initial step towards a rigorous and contract-driven methodology for the use of machine learning in the automotive domain. This is largely due to the alignment between the tolerance as regulated in the contract (specification) and the corresponding tolerance-integrated loss function (architecture design). Although the work is motivated by concrete problems in direct perception, the underlying technique can be applicable to other applications in automated driving where regression is used.

For future work, we plan to apply similar concepts to other domains such as medical diagnosis, as well as developing analogous concepts for recurrent neural networks. Within the automotive domain, we also plan to bring our proposed contract-based approach for machine learning to standardization bodies, with the goal of revising existing autonomous driving safety standards such as ISO $21448^{7}$

\section{REFERENCES}

[1] D. A. Pomerleau, "Alvinn: An autonomous land vehicle in a neural network," in Advances in Neural Information Processing Systems (NIPS), 1989, pp. 305-313.

[2] C. Chen, A. Seff, A. Kornhauser, and J. Xiao, "DeepDriving: Learning affordance for direct perception in autonomous driving," in Proceedings of the International Conference on Computer Vision (ICCV). IEEE, 2015, pp. 2722-2730.

[3] A. Sauer, N. Savinov, and A. Geiger, "Conditional affordance learning for driving in urban environments," in Proceedings of the 2nd Conference on Robot Learning (CoRL). PMLR, 2018, pp. 237-252.

[4] E. Wong and Z. Kolter, "Provable defenses against adversarial examples via the convex outer adversarial polytope," in Proceedings of the 35th international conference on International Conference on Machine Learning (ICML), 2018, pp. 5283-5292.

[5] A. Sinha, H. Namkoong, and J. C. Duchi, "Certifying some distributional robustness with principled adversarial training," in Proceedings of the 6th International Conference on Learning Representations (ICLR), 2018.

[6] S. Wang, Y. Chen, A. Abdou, and S. Jana, "Mixtrain: Scalable training of formally robust neural networks," arXiv preprint arXiv:1811.02625 2018.

[7] A. Raghunathan, J. Steinhardt, and P. Liang, "Certified defenses against adversarial examples," in Proceedings of the 6th International Conference on Learning Representations (ICLR), 2018.

[8] E. Wong, F. Schmidt, J. H. Metzen, and J. Z. Kolter, "Scaling provable adversarial defenses," in Advances in Neural Information Processing Systems (NIPS), 2018, pp. 8400-8409.

${ }^{7}$ SOTIF: https://www.iso.org/standard/70939.html 
[9] Y. Tsuzuku, I. Sato, and M. Sugiyama, "Lipschitz-margin training: Scalable certification of perturbation invariance for deep neural networks," in Advances in Neural Information Processing Systems (NIPS), 2018, pp. 6541-6550.

[10] H. Salman, G. Yang, J. Li, P. Zhang, H. Zhang, I. Razenshteyn, and S. Bubeck, "Provably robust deep learning via adversarially trained smoothed classifiers," arXiv preprint arXiv:1906.04584 2019.

[11] V. Nair and G. E. Hinton, "Rectified linear units improve restricted boltzmann machines," in Proceedings of the 27th international conference on machine learning (ICML), 2010, pp. 807-814.

[12] D. Clevert, T. Unterthiner, and S. Hochreiter, "Fast and accurate deep network learning by exponential linear units (elus)," in Proceedings of the 4th International Conference on Learning Representations (ICLR), 2016.

[13] A. L. Maas, A. Y. Hannun, and A. Y. Ng, "Rectifier nonlinearities improve neural network acoustic models," in Proceedings of the 30th International Conference on Machine Learning (ICML), vol. 30, no. 1, 2013, p. 3.

[14] P. Cousot and R. Cousot, "Abstract interpretation: a unified lattice model for static analysis of programs by construction or approximation of fixpoints," in Proceedings of the 4th ACM SIGACT-SIGPLAN symposium on Principles of Programming Languages (POPL). ACM, 1977, pp. 238-252.

[15] C.-H. Cheng, G. Nührenberg, and H. Ruess, "Maximum resilience of artificial neural networks," in Proceedings of the 15th International Symposium on Automated Technology for Verification and Analysis (ATVA). Springer, 2017, pp. 251-268.

[16] X. Glorot and Y. Bengio, "Understanding the difficulty of training deep feedforward neural networks," in Proceedings of the 13th international conference on artificial intelligence and statistics (AISTATS), 2010, pp. 249-256.

[17] D. P. Kingma and J. Ba, "Adam: A method for stochastic optimization," arXiv preprint arXiv:1412.6980 2014.

[18] C. Szegedy, W. Zaremba, I. Sutskever, J. Bruna, D. Erhan, I. Goodfellow, and R. Fergus, "Intriguing properties of neural networks," arXiv preprint arXiv:1312.6199 2013.

[19] T. Gehr, M. Mirman, D. Drachsler-Cohen, P. Tsankov, S. Chaudhuri, and M. Vechev, "Ai2: Safety and robustness certification of neural networks with abstract interpretation," in Proceedinfs of the 2018 IEEE Symposium on Security and Privacy (Oakland). IEEE, 2018, pp. 318.

[20] N. Akhtar and A. Mian, "Threat of adversarial attacks on deep learning in computer vision: A survey," IEEE Access, vol. 6, pp. 14 410-14 430, 2018

[21] K. Pei, Y. Cao, J. Yang, and S. Jana, "Deepxplore: Automated whitebox testing of deep learning systems," in Proceedings of the 26th Symposium on Operating Systems Principles (SOSP). ACM, 2017, pp. 1-18.

[22] Y. Sun, X. Huang, and D. Kroening, "Testing deep neural networks," arXiv preprint arXiv:1803.04792 2018

[23] Y. Sun, M. Wu, W. Ruan, X. Huang, M. Kwiatkowska, and D. Kroening, "Concolic testing for deep neural networks," arXiv preprint arXiv:1805.00089. 2018.

[24] C. Cheng, C. Huang, and H. Yasuoka, "Quantitative projection coverage for testing ml-enabled autonomous systems," in Proceedings of the 16th International Symposium on Automated Technology for Verification and Analysis (ATVA). Springer, 2018, pp. 126-142.

[25] L. Pulina and A. Tacchella, "An abstraction-refinement approach to verification of artificial neural networks," in Proceedings of the 22th International Conference on Computer Aided Verification (CAV). Springer, 2010, pp. 243-257.

[26] G. Katz, C. W. Barrett, D. L. Dill, K. Julian, and M. J. Kochenderfer, "Reluplex: An efficient SMT solver for verifying deep neural networks," in Proceedings of the 29th International Conference on Computer Aided Verification (CAV). Springer, 2017, pp. 97-117.

[27] X. Huang, M. Kwiatkowska, S. Wang, and M. Wu, "Safety verification of deep neural networks," in Proceedings of the 29th International Conference on Computer Aided Verification (CAV), 2017, pp. 3-29.

[28] R. Ehlers, "Formal verification of piece-wise linear feed-forward neural networks," in Proceedings of the 15th International Symposium on Automated Technology for Verification and Analysis (ATVA). Springer, 2017, pp. 269-286.

[29] A. Lomuscio and L. Maganti, "An approach to reachability analysis for feed-forward ReLU neural networks," arXiv preprint arXiv:1706.07351. 2017.
[30] N. Narodytska, S. Kasiviswanathan, L. Ryzhyk, M. Sagiv, and T. Walsh, "Verifying properties of binarized deep neural networks," in Proceedings of the 32nd AAAI Conference on Artificial Intelligence, 2018, pp. 6615-6624.

[31] S. Dutta, S. Jha, S. Sankaranarayanan, and A. Tiwari, "Output range analysis for deep feedforward neural networks," in Proceedings of the 11th International Symposium on NASA Formal Methods (NFM). Springer, 2018, pp. 121-138.

[32] R. R. Bunel, I. Turkaslan, P. Torr, P. Kohli, and P. K. Mudigonda, "A unified view of piecewise linear neural network verification," in Advances in Neural Information Processing Systems (NIPS), 2018, pp. $4795-4804$.

[33] W. Ruan, X. Huang, and M. Kwiatkowska, "Reachability analysis of deep neural networks with provable guarantees," in Proceedings of the 27th International Joint Conference on Artificial Intelligence (IJCAI), 2018, pp. 2651-2659.

[34] S. Wang, K. Pei, J. Whitehouse, J. Yang, and S. Jana, "Formal security analysis of neural networks using symbolic intervals," in Proceedings of the 27th USENIX Security Symposium (USENIX), 2018, pp. 15991614.

[35] T.-W. Weng, H. Zhang, H. Chen, Z. Song, C.-J. Hsieh, D. Boning, I. S. Dhillon, and L. Daniel, "Towards fast computation of certified robustness for ReLU networks," in Proceedings of the 35th international conference on International Conference on Machine Learning (ICML), 2018, pp. 5276-5285.

[36] P. Yang, J. Liu, J. Li, L. Chen, and X. Huang, "Analyzing deep neural networks with symbolic propagation: Towards higher precision and faster verification," arXiv preprint arXiv:1902.09866 2019.

[37] C. Huang, J. Fan, W. Li, X. Chen, and Q. Zhu, "ReachNN: Reachability analysis of neural-network controlled systems," arXiv preprint arXiv:1906.10654 2019.

[38] K. Dvijotham, R. Stanforth, S. Gowal, T. Mann, and P. Kohli, "A dual approach to scalable verification of deep networks," arXiv preprint arXiv:1803.06567. 2018. 